\title{
Convalescent plasma successfully treats a severe COVID-19 patient with multi-organ failure
}

\author{
Behnam Bajelan ${ }^{1}$, Pejman Salehifar ${ }^{2}$, Afshin Karami ${ }^{3}$, Mehdi Salimi ${ }^{4}$, Alireza Janbakhsh ${ }^{5}$, Zahra Maleki ${ }^{1}$, \\ Khosro Farhadi ${ }^{1}$, Behnaz Karimi ${ }^{1}$, Mohammad Tahmasebi ${ }^{6}$, Afshin Ghasemi ${ }^{7}$, Ali Gholami ${ }^{1}$, Babak Sayad ${ }^{5}$, \\ Mehrdad Payandeh ${ }^{8}$ **
}

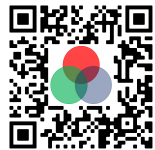

Use your smartphone to scan this QR code and download this article

${ }^{1}$ Department of Anesthesiology, Kermanshah University of Medical Sciences. Kermanshah, Iran

${ }^{2}$ Department of Biotechnology, Kermanshah Blood Transfusion Organization.

Kermanshah, Iran

${ }^{3}$ Department of Hematology, Regenerative Medicine Research Center, Kermanshah University of Medical Sciences. Kermanshah, Iran

${ }^{4}$ Department of Pulmonary, Kermanshah University of Medical Sciences. Kermanshah, Iran

Correspondence

Mehrdad Payandeh, Department of Hematology and Medical Oncology, Regenerative Medicine Research Center, Kermanshah University of Medical Sciences. Kermanshah, Iran

Email: mpayandeh72@yahoo.com History

- Received: Aug 30, 2020

- Accepted: Sep 23, 2020

- Published: Oct 04, 2020

DOI : 10.15419/bmrat.v7i10.635

\section{Check for updates}

\section{Copyright}

(c) Biomedpress. This is an openaccess article distributed under the terms of the Creative Commons Attribution 4.0 International license.

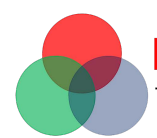

\begin{abstract}
Due to the high mortality rate of coronavirus disease 2019 (COVID-19) and the lack of specific treatment for the disease, it is essential to find new therapies. The present case report aimed to assess the efficiency of convalescent plasma in patients with severe acute respiratory syndrome coronavirus 2. We reported a severe case of COVID-19 with multi-organ failure, who had reduced oxygen saturation after several courses of antiviral therapy. Moreover, computed tomography (CT) scan results showed patchy lesions in the base of lungs. Therapeutic measures, including endotracheal intubation and plasmapheresis with convalescent plasma, were performed for the patient; subsequently, good responses to the treatments were observed. Our findings demonstrate that convalescent plasma improves pulmonary lesions and the patient's clinical status.

Key words: COVID-19, severe acute respiratory syndrome coronavirus 2, convalescent plasma,
\end{abstract} plasmapheresis

\section{INTRODUCTION}

In December 2019, the first case of severe acute respiratory syndrome (SARS) caused by the novel coronavirus disease 2019 (COVID-19) was identified in Wuhan, the capital city of Hubei, China ${ }^{1}$. The disease spread so rapidly around the globe that the World Health Organization (WHO) declared COVID-19 a pandemic in late March 2020. By June 2020, COVID19 had been confirmed in 8.03 million people and had claimed 436,900 lives worldwide ${ }^{2}$. COVID-19 is an enveloped RNA beta-coronavirus, which is now termed as severe acute respiratory syndrome coronavirus 2 (SARS-CoV-2) because of its phylogenetic similarity to severe acute respiratory syndrome coronavirus (SARS-CoV) ${ }^{3,4}$. COVID-19 causes severe acute respiratory illness and is associated with a high mortality rate ${ }^{5}$. Currently, there is no definitive cure for COVID-19 infection, and global efforts have been intensified to discover new therapies. Convalescent plasma (CP) therapy is one of the recently identified therapies which may be helpful in the treatment of SARS-CoV-2 ${ }^{6,7}$. This study aimed to describe the first clinical experience with CP transfusion administered to severely ill patients with COVID-19 in Kermanshah, Iran.

\section{CASE PRESENTATION}

On March 12, 2020, a 58-year-old man with a fever of $38^{\circ} \mathrm{C}$ and history of cardiorespiratory and kidney failure was admitted to the Imam Reza Hospital, Kermanshah, Iran. He was monitored for seven days and received oral antibiotics. After this period, his temperature returned to normal, but he was suffering from dyspnea, headache and confusion. The patient's C-reactive protein test was positive, and a computed tomography (CT) scan was performed immediately. The CT s can results demonstrated bilateral groundglass opacities in the patient's lungs. Further, primary laboratory investigations showed that he had leukocytosis (WBC: $18,400 / \mathrm{mm}^{3}$ ) with severe lymphopenia (5\%). He also experienced an increase in blood urea nitrogen (BUN) and serum creatinine levels (BUN 64 $\mathrm{mg} / \mathrm{dL}, \mathrm{Cr} 1.8 \mathrm{mg} / \mathrm{dL}$ ), indicating the symptoms of kidney failure. On admission, his arterial blood gas analysis indicated the following: $\mathrm{PH} 7.476 \uparrow, \mathrm{PCO}_{2}$ $42.1 \mathrm{mmHg} \downarrow, \mathrm{PO}_{2} 37.0 \mathrm{mmHg} \downarrow$, and $\mathrm{HCO}_{3}-31.61$ $\mathrm{mmol} / \mathrm{L} \downarrow$, with blood oxygen saturation of $92 \%$.

Based on the results of routine tests and chest CT findings, the patient was admitted to an airborne-isolation unit as a suspected case of COVID-19. Antiviral treatments including Hydroxychloroquine (200 mg BID per day), Kaletra (2 tab/BID), and supportive care were used. Moreover, Imipenem ( $0.5 \mathrm{~g}$ every 
${ }^{5}$ Department of Infectious Diseases and Tropical Medicine, Kermanshah University of Medical Sciences. Kermanshah, Iran

${ }^{6}$ Department of Laboratory Sciences, Kermanshah University of Medical Sciences. Kermanshah, Iran

${ }^{7}$ Department of General Surgery, Kermanshah University of Medical Sciences. Kermanshah, Iran

${ }^{8}$ Department of Hematology and Medical Oncology, Regenerative Medicine Research Center, Kermanshah University of Medical Sciences. Kermanshah, Iran
$12 \mathrm{~h}$, intravenously) and Targocid (0.4 g q.d, intravenously) were used in the treatment of the disease. The result of the pharyngeal s wab for SARS-CoV-2 using the qualitative real-time reverse-transcriptasepolymerase-chain-reaction (Quest SARS-CoV-2 rRTPCR) assay was positive 72 hours after admission. Combining all the laboratory results with full discussion, we confirmed SARS-CoV-2 infection in the patient. On March 23, due to severe respiratory distress and reduced oxygen saturation $\left(\mathrm{SpO}_{2}<75 \%\right)$ in the patient, endotracheal intubation was performed to assist breathing. After intubation for four d ays, oxygen saturation reached a normal range (94.2\%). A CP request was sent following a Kermanshah-Iran blood bank's announcement that antibodies in COVID-19 recovered plasma could help treat these patients. According to the New York Times, convalescent plasma might contain valuable proteins that can reduce inflammation in patients with COVID-19 within 12 to 24 hours.

The two d onors of convalescent plasma who recovered from SARS-CoV-2 infection were invited to donate their convalescent plasma. The mean donor age in this study was less than 40 years old. After testing negative for SARS-CoV-2, hepatitis B virus, hepatitis $\mathrm{C}$ virus, HIV and syphilis at the time of blood donation, the donors were invited to donate their CP. The patient's blood group was determined for adaptability with the $\mathrm{CP}$ donors, and apheresis was performed with a cell separator. Following this, $1500 \mathrm{~mL}$ of convalescent plasma was obtained from the donors over three sessions. At each session, the patient received $500 \mathrm{~mL}$ of convalescent plasma. Therefore, the patient received $\mathrm{CP}$ from compatible donors during the three plasmapheresis sessions (days 3, 5 and 7 after admission), as an adjunctive treatment during septic shock. As clearly shown in Figure 1, this treatment reduced the count of activated blood leukocytes and increased the count of blood lymphocytes. Moreover, renal function tests showed a relative improvement in renal failure with the following results: BUN: $37 \mathrm{mg} / \mathrm{dL}$ and Cr $1.5 \mathrm{mg} / \mathrm{dL}$. These results are consistent with absorption of pulmonary lesions and increased arterial oxygen saturation after plasma transfusion (Figure 2). Hence, CP therapy can be a potentially effective treatment in SARS-CoV-2-infected patients.

\section{DISCUSSION}

Antibodies are proteins made by the immune system to fight invaders, such as viruses, bacteria, or other foreign substances ${ }^{5}$. In the past, the use of pooled plasma from patients recovering from West Nile encephalitis had good clinical effects on infected patients ${ }^{6}$. Moreover, CP was used as a last resort to improve the survival of SARS patients with acute conditions ${ }^{7}$. Given the recommendation of the $\mathrm{WHO}$ and the efficacy of this method in improving the condition of patients with Ebola virus disease and Middle East respiratory syndrome coronavirus, convalescent plasma is likely to be helpful in the treatment of COVID-19 patients ${ }^{8,9}$. Furthermore, on February 8, the China National Biotec Group Co. claimed in a statement on its official WeChat account that they used this plasma (containing highly potent antibodies) to treat more than 10 seriously ill patients. The company claimed that those receiving the treatment improved within 24 hours, with reduced inflammation and viral loads, as well as better oxygen levels in the blood ${ }^{10}$.

However, people who have recently recovered from COVID-19 still have antibodies to coronavirus circulating in their blood. Injecting these antibodies into patients can theoretically help them better fight the infection. Therefore, in our intensive care unit (ICU), the patient was treated with three sessions of plasmapheresis. A good response to the therapy was observed in that convalescent plasma improved pulmonary lesions, followed by decreased levels of activated blood leukocytes, and recovery of the patient without sequelae. Another outcome was the improvement of renal failure after the three stages of $\mathrm{CP}$ therapy- on days 3, 5 and 7. Interestingly, no complications associated with plasma infusion were observed. This treatment transfers the immunity from a recovered patient to a sick patient, as an approach previously used in flu pandemics. Since there is no fully licensed treatment or vaccine against SARS-CoV-2 and because the process of producing and testing drugs can take months or even years, the use of $\mathrm{CP}$ can be the most effective treatment for the infection to significantly reduce mortality rates.

\section{CONCLUSION}

We used CP therapy on a COVID-19 patient for the first time in Iran and, fortunately, observed positive results. Thus, this treatment can play a helpful role in the treatment of severe sepsis caused by COVID-19 and immune complex-mediated organ injury. However, a study with a higher number of cases and controls is needed to examine the effectiveness of this treatment in greater detail. 


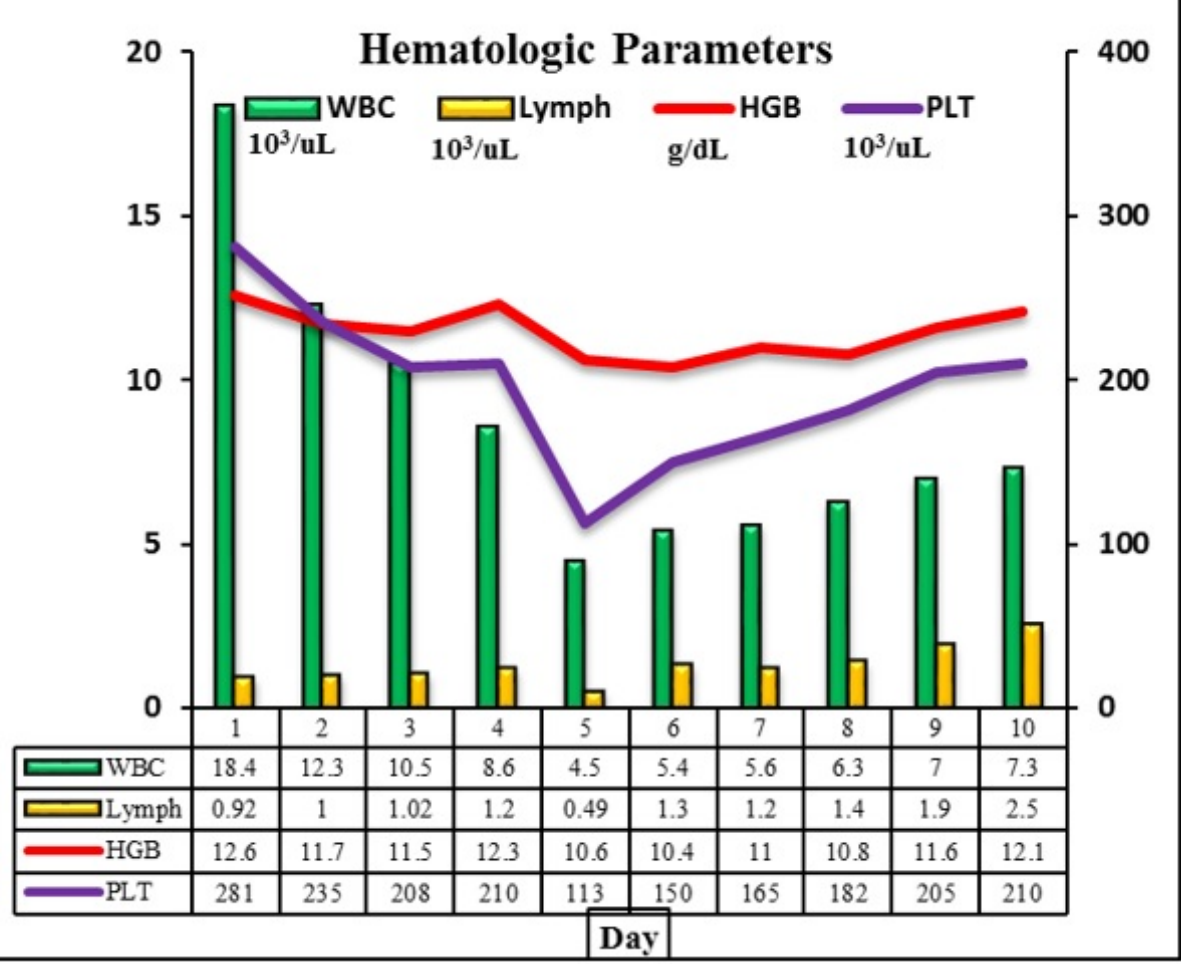

Figure 1: Changes of hematologic parameters relative to day of illness and CP. CP therapy reduced the count of activated blood leukocytes and increased the count of blood lymphocytes.

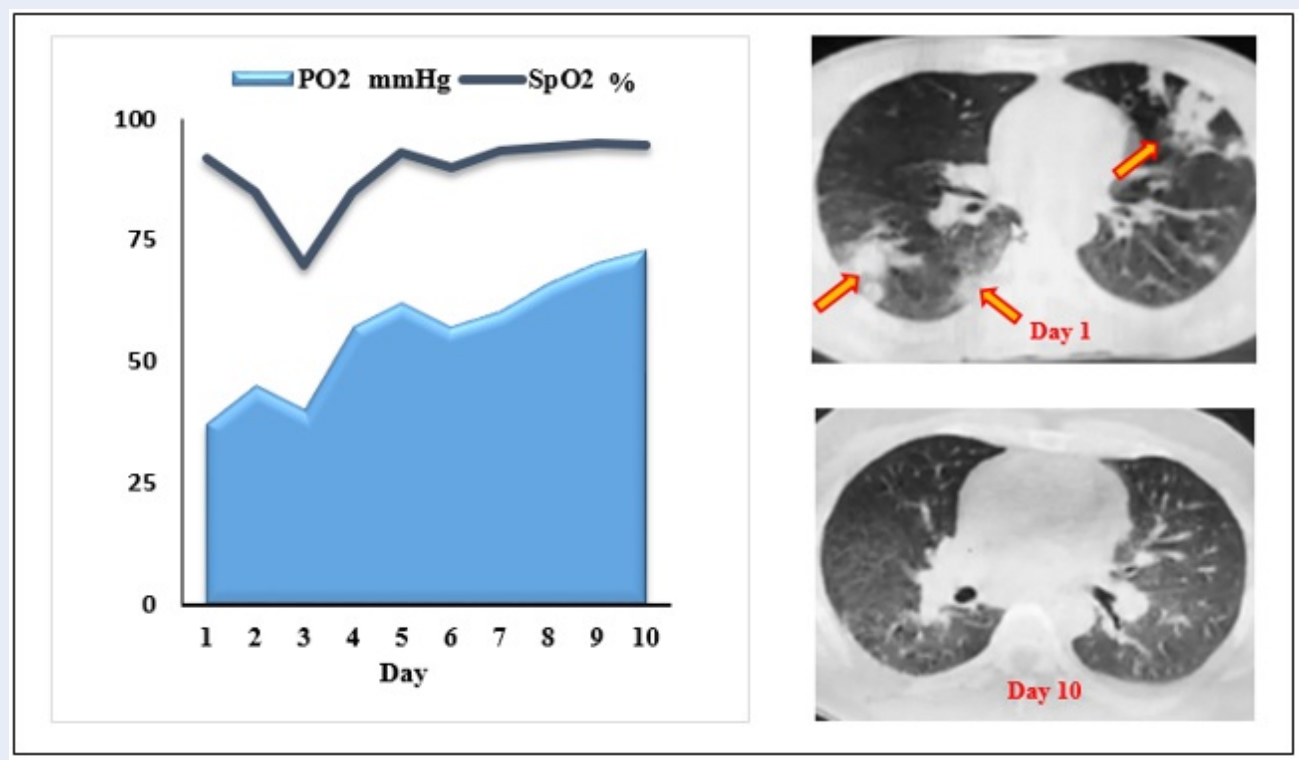

Figure 2: Changes in $\mathrm{SpO}_{2}$ and lung $\mathrm{CT}$ scan during $\mathrm{CP}$ therapy. $\mathrm{CP}$ therapy improved pulmonary lesions and increased arterial oxygen saturation. 


\section{ABBREVIATIONS}

BUN: Blood Urea Nitrogen

COVID-19: coronavirus disease 2019

CP: Convalescent Plasma

SARS-CoV-2: Severe Acute Respiratory Syndrome Coronavirus 2

WHO: World Health Organization

\section{ACKNOWLEDGMENTS}

Not applicable.

\section{AUTHOR'S CONTRIBUTIONS}

Mehrdad Payandeh, Behnam Bajelan, Pejman Salehifar \& Alireza Janbakhsh: Literature search, Clinical studies, Data acquisition, Data analysis; Zahra Maleki \& Afshin Karami: Manuscript preparation, Manuscript review, Guarantor; Babak Sayad, Khosro Farhadi, Behnaz Karimi, Mohammad Tahmasebi \&, Afshin Ghasemi: Concepts, Design, Definition of intellectual content, Literature search, Manuscript editing; Ali Gholami \& Afshin Karami: Manuscript editing, Manuscript preparation, Literature search. All authors read and approved the final manuscript.

\section{FUNDING}

Not applicable.

\section{AVAILABILITY OF DATA AND MATERIALS}

Data and materials used and/or analyzed during the current study are available from the corresponding author on reasonable request.

\section{ETHICS APPROVAL AND CONSENT TO PARTICIPATE}

The Medical Ethics Research Committee of Imam Reza Hospital, Kermanshah, Iran, approved the study, and all participants provided written informed consent.

\section{CONSENT FOR PUBLICATION}

Not applicable.

\section{COMPETING INTERESTS}

The authors declare that they have no competing interests.

\section{REFERENCES}

1. Guan WJ, et al. Clinical characteristics of coronavirus disease 2019 in China. New England journal of medicine. 2020;382(18):1708-1720. PMID: 32109013. Available from: https://doi.org/10.1056/NEJMoa2002032.

2. Ellinghaus D, Degenhardt F, Bujanda L, Buti M, Albillos A, et al. Genomewide Association Study of Severe Covid-19 with Respiratory Failure. New England Journal of Medicine. 2020;2(5):479-571. Available from: https://doi.org/10.1056/ NEJMoa2020283.

3. Wu D, Wu T, Liu Q, Yang Z. The SARS-CoV-2 outbreak: what we know. International Journal of Infectious Diseases. 2020;94:44-48. PMID: 32171952. Available from: https://doi. org/10.1016/j.ijid.2020.03.004.

4. Chen $\mathrm{L}$, et al. Convalescent plasma as a potential therapy for COVID-19. The Lancet Infectious Diseases. 2020;20(4):398400. Available from: https://doi.org/10.1016/S1473-3099(20) 30141-9.

5. Janeway C, et al. Part II: recognition of antigen. Immunobiology, 5th ed, Garland Science, New York and London. 2001;.

6. Ben-Nathan D, et al. Prophylactic and therapeutic efficacy of human intravenous immunoglobulin in treating West Nile virus infection in mice. The Journal of infectious diseases. 2003;188(1):5-12. PMID: 12825165. Available from: https: //doi.org/10.1086/376870.

7. Soo $Y$, et al. Retrospective comparison of convalescent plasma with continuing high-dose methylprednisolone treatment in SARS patients. Clinical microbiology and infection. 2004;10(7):676-678. PMID: 15214887. Available from: https: //doi.org/10.1111/j.1469-0691.2004.00956.x.

8. Florescu DF, et al. Administration of brincidofovir and convalescent plasma in a patient with Ebola virus disease. Clinical Infectious Diseases. 2015;61(6):969-973. PMID: 25991468. Available from: https://doi.org/10.1093/cid/civ395.

9. Arabi Y. Feasibility, safety, clinical, and laboratory effects of convalescent plasma therapy for patients with Middle East respiratory syndrome coronavirus infection: a study protocol. Springerplus. 2015;4(1):1-8. PMID: 26618098. Available from: https://doi.org/10.1186/s40064-015-1490-9.

10. China Seeks Plasma From Recovered Patients as Virus Treatment. Time. 2020;. 